\title{
Algorithms for Switching Between Boolean and Arithmetic Masking of Second Order
}

\author{
Praveen Kumar Vadnala and Johann Großschädl \\ University of Luxembourg, \\ Laboratory of Algorithmics, Cryptology and Security (LACS), \\ 6, rue Richard Coudenhove-Kalergi, L-1359 Luxembourg \\ \{praveen.vadnala, johann.groszschaedl\}@uni.lu
}

\begin{abstract}
Masking is a widely-used countermeasure to thwart Differential Power Analysis (DPA) attacks, which, depending on the involved operations, can be either Boolean, arithmetic, or multiplicative. When used to protect a cryptographic algorithm that performs both Boolean and arithmetic operations, it is necessary to change the masks from one form to the other in order to be able to unmask the secret value at the end of the algorithm. To date, known techniques for conversion between Boolean and arithmetic masking can only resist first-order DPA. This paper presents the first solution to the problem of converting between Boolean and arithmetic masking of second order. To set the context, we show that a straightforward extension of first-order conversion schemes to second order is not possible. Then, we introduce two algorithms to convert from Boolean to arithmetic masking based on the second-order provably secure S-box output computation method proposed by Rivain et al (FSE 2008). The same can be used to obtain second-order secure arithmetic to Boolean masking. We prove the security of our conversion algorithms using similar arguments as Rivain et al. Finally, we provide implementation results of the algorithms on three different platforms.
\end{abstract}

Keywords: Differential power analysis, Second-order DPA, Arithmetic masking, Boolean Masking, Provably secure masking

\section{Introduction}

Side-channel cryptanalysis exploits information leakage from the execution of a concrete implementation of a cryptographic algorithm [12]. Therefore, this kind of attack is methodically very different from "traditional" cryptanalysis, which essentially focuses on finding secret keys in a black box model given only pairs of plaintexts and ciphertexts. The first form of side-channel attacks discussed in the literature are timing attacks, i.e. attacks exploiting measurable differences in the execution time of a cryptographic algorithm or a specific operation it is based upon $[13,11]$. A more sophisticated class of attacks are power analysis attacks, which aim to deduce information about the secret key from the power consumption of the device while a certain operation is carried out [15]. A third 
class are electromagnetic (EM) attacks, which exploit the relationship between secret data and EM emanations produced by the device [1].

Power analysis attacks received extensive attention from the cryptographic community ever since Kocher and his team published them for the first time in their seminal paper Differential Power Analysis [14]. These attacks allow one to recover the full secret key with relatively few measurements and it is close to impossible to totally circumvent them with current semiconductor technologies [15]. While Simple Power Analysis (SPA) attacks try to recover a secret value by directly "comparing" the power measurements with the corresponding operations, Differential Power Analysis (DPA) attacks are much more sophisticated and aim to reveal a secret value by applying statistical techniques on multiple measurements of the same operation. Masking is a widely-used countermeasure to thwart DPA attacks, which involves using random variables, called masks, to reduce the correlation between the secret value and the obtained leakage [2]. In order to circumvent first-order DPA attacks [15] that involve a single operation using masking, we divide the secret value into two shares: a mask generated randomly and the masked value of the secret. However, this approach can still be attacked via a second-order DPA involving two operations corresponding to the two shares of the secret $[16,19]$. In general, a $d$-th order masking scheme is vulnerable to a $(d+1)$-th order DPA attack involving all $d+1$ shares of the secret. These attacks are called Higher-Order DPA attacks (HODPA).

Depending on the operation to be protected, a masking scheme can either be Boolean (using logical XOR), arithmetic (using modular addition/subtraction) or multiplicative (using modular multiplication). To successfully "unmask" the variable at the end of the algorithm, one has to track the change of the masked secret value during the execution of the algorithm. If an algorithm contains two of the three afore-mentioned operations (i.e. XOR, modular addition/subtraction, modular multiplication), the masks have to be converted from one form to the other, keeping this conversion free from any leakage. Goubin introduced secure methods to convert between first-order Boolean and arithmetic masks in [10]. Coron and Tchulkine improved the method for switching from arithmetic to Boolean masking in [5], which was recently further improved by Debraize in [6]. While solutions exist for converting between arithmetic and multiplicative masking of higher order [7-9], the conversion between Boolean and arithmetic masking is currently limited to first order. We aim to fill this gap by presenting algorithms to switch between Boolean and arithmetic masks of second order.

In the context of second-order masking, a sensitive variable $x$ is represented by three shares; these are $x_{1}=x \oplus x_{2} \oplus x_{3}, x_{2}$ and $x_{3}$ in the case of Boolean masking, and $A_{1}=x-A_{2}-A_{3}, A_{2}$ and $A_{3}$ for arithmetic masking [15]. The problem is to convert between Boolean and arithmetic masking without introducing any first-order or second-order leakage. Unfortunately, it is not possible to extend the existing first-order secure conversion schemes to second order as we will show later. Therefore, we employ techniques proposed by Rivain et al in [21] to arrive at the first solution for converting second-order masks from one form to the other. In [21], the authors describe two provably secure methods to 
compute S-box outputs without first or second-order leakage. By applying these methods, we present a total of four algorithms, two for each conversion type.

The rest of the paper is organized as follows. We review some of the existing solutions for a first-order conversion as well as the paper of Rivain et al in Section 2. Then, we show that a straightforward application of Goubin's method is insecure for second-order conversion in Section 3. We introduce our algorithms for Boolean to arithmetic conversion in Section 4. The algorithms to convert in the opposite direction (i.e. arithmetic to Boolean) can be derived similarly and are described in Section 5. We prove the security of our algorithms in Section 6 for a device leaking in the Hamming weight model. Section 7 summarizes some implementation results and, finally, Section 8 concludes the paper.

\section{Previous Work}

This section provides an overview of recent results that will be used later in the paper. We first summarize existing methods for switching between arithmetic and Boolean masking of first order. Then, we describe two techniques proposed by Rivain et al at FSE 2008 to compute S-box outputs secure against secondorder DPA attacks [21].

\subsection{Securing Conversions Against First-Order DPA}

The first solution to the problem of Boolean to arithmetic mask conversion was presented by Messerges in [17], which was later proven to be insecure by Coron and Goubin in [4]. At CHES 2001, Goubin proposed an algorithm for switching between Boolean and arithmetic masks secure against first-order DPA attacks [10]. His algorithm to convert from Boolean to arithmetic masking is based on the following fact: for $I=\left\{0,1, \ldots, 2^{n}-1\right\}$ with $n \geq 1$ and $x^{\prime} \in I$, the function $\phi_{x^{\prime}}(r): I \rightarrow I$ defined as $\phi_{x^{\prime}}(r)=\left(x^{\prime} \oplus r\right)-r \bmod 2^{n}$ is affine over the field $\mathrm{GF}(2)$. Therefore, the function $\psi_{x^{\prime}}=\phi_{x^{\prime}} \oplus \phi_{x^{\prime}}(0)$ is linear over GF(2) and, as a result, $x=x^{\prime} \oplus r$ with Boolean shares $\left(x^{\prime}, r\right)$ can be converted to the equivalent arithmetic shares $(A, r)$ for any random $\gamma$ via the following relation:

$$
\begin{aligned}
A & =\phi_{x^{\prime}}(r)=\psi_{x^{\prime}}(r) \oplus x^{\prime} \\
& =\psi_{x^{\prime}}(\gamma) \oplus \psi_{x^{\prime}}(r \oplus \gamma) \oplus x^{\prime} \\
& =\left[\left(x^{\prime} \oplus \gamma\right)-\gamma\right] \oplus x^{\prime} \oplus\left[\left(x^{\prime} \oplus(r \oplus \gamma)\right)-(r \oplus \gamma)\right]
\end{aligned}
$$

This method is highly efficient, requiring only a constant number of elementary operations. Goubin's arithmetic to Boolean conversion is based on the following fact: $x^{\prime}=(A+r) \oplus r$ is equivalent to $x^{\prime}=A \oplus u_{n-1}$, where

$$
\left\{\begin{array}{l}
u_{0}=0 \\
u_{i+1}=2\left[u_{i} \wedge(A \oplus r) \oplus(A \wedge r)\right] \forall i \geq 0
\end{array}\right.
$$

Unfortunately, this method is far less efficient since the number of operations is proportional to the size of the registers. 


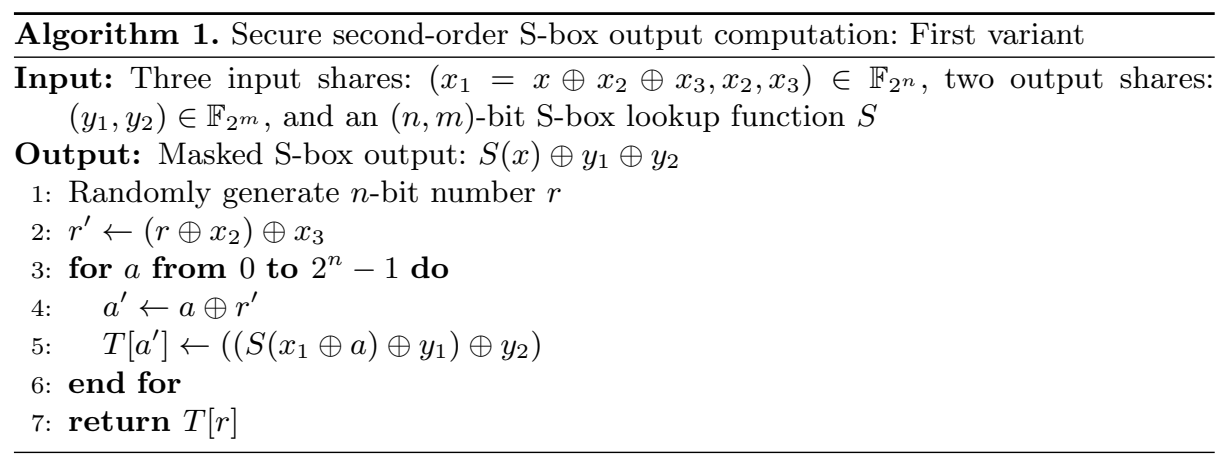

In 2003, Coron and Tchulkine proposed a new algorithm for conversion from arithmetic to Boolean masking [5]. To convert two $n$-bit $(n=p \cdot k)$ arithmetic shares $A$ and $R$ with $x=A+R \bmod n$ into two Boolean shares $x^{\prime}$ and $R$ such that $x=x^{\prime} \oplus R$, the algorithm works on each $k$-bit word independently. Therefore, $A$ and $R$ are divided into $p$ words of $k$ bits: $A=A_{1}\left\|A_{2}\right\| \ldots \| A_{p-1}$ and $R=R_{1}\left\|R_{2}\right\| \ldots \| R_{p-1}$. Now, the Boolean share equivalent to the $i$-th word of the arithmetic share $A_{i}$ is computed as $x_{i}^{\prime}=\left(A_{i}+R_{i}+c_{i+1}\right) \oplus R_{i}$, where $c_{i+1}$ is the carry bit produced from the previous $k$-bit word. The algorithm precomputes two small tables of $2^{k}$ entries each, and reuses them several times in the course of the conversion. The first table serves to convert each arithmetic-share word independently to the equivalent Boolean-share word. This table contains the entries $(z+r) \oplus r$ for all possible values of $z \in\left[0,2^{k}-1\right]$ and the random value $r \in\left[0,2^{k}-1\right]$. The correct value for the $i$-th word can be obtained when $z=\left(A_{i}-r+R_{i}\right)+c_{i+1}$. The second table is used to mask the carry that needs to be passed from one word to the next-higher. Even though this increases the memory requirements, the conversion time is reduced significantly. Neiße and Pulkus [18] modified the algorithm so as to reduce the memory needed to store the tables. At CHES 2012, Debraize discovered a bug in the Coron-Tchulkine algorithm and devised a new variant that is also more efficient [6].

\subsection{Generic Countermeasure Against Second-Order DPA}

At FSE 2008, Rivain et al proposed two algorithms to protect the computation of S-box outputs against second-order attacks [21]. Given three input shares of a secret value $x$, namely $x_{1}=x \oplus x_{2} \oplus x_{3}, x_{2}$, and $x_{3}$ (which are all in $\mathbb{F}_{2^{n}}$ ) and two output shares $y_{1}, y_{2} \in \mathbb{F}_{2^{m}}$ along with an $(n, m)$ S-box lookup function $S$, they compute the third share $y_{3}$ such that $y_{1} \oplus y_{2} \oplus y_{3}=S(x)$. Hence, we have $y_{3}=S(x) \oplus y_{1} \oplus y_{2}$. The algorithms compute $\left(S\left(x_{1} \oplus a\right) \oplus y_{1}\right) \oplus y_{2}$ for all possible values of $a$ (i.e. $0 \leq a \leq 2^{n-1}$ ), among which the correct value can be obtained when $a=x_{2} \oplus x_{3}$. We recall these algorithms below.

Algorithm 1 uses a table of $2^{n}$ entries to store $\left(S\left(x_{1} \oplus a\right) \oplus y_{1}\right) \oplus y_{2}$ for all possible values of $a$. Here, the value $\left(x_{2} \oplus x_{3}\right)$ is masked via a random variable $r$, the result of which is assigned to $r^{\prime}$. Thereafter, the entry corresponding to 


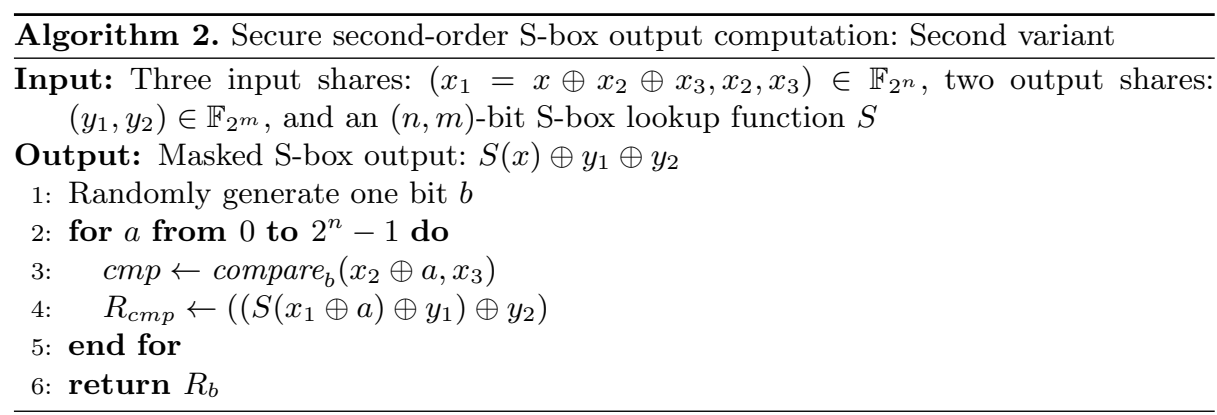

$\left(S\left(x_{1} \oplus a\right) \oplus y_{1}\right) \oplus y_{2}$ will be stored at location $a^{\prime}=a \oplus r^{\prime}$. The correct value of the third share $y_{3}$ can be retrieved by accessing the value stored in the table at location $T[r]$. As $r=a^{\prime}$, the value of $a$ becomes $a=r \oplus r^{\prime}=x_{2} \oplus x_{3}$, thus yielding the desired result.

The security of Algorithm 1 can be proven by showing that it is impossible to recover $x$ by combining any pair of intermediate variables computed by the algorithm. We refer the interested reader to Section 3.1 in [21] for the complete proof. In Section 6, we will use the same approach to prove the security of our conversion techniques. Algorithm 1 requires a table of $2^{n}$ words (each having a length of $m$ bits) in RAM, and is, therefore, not suitable for low-cost devices. To overcome this issue, Rivain et al introduced another algorithm consuming less memory at the expense of executing more operations.

Algorithm 2 specifies the second solution proposed by Rivain et al in [21] to securely compute an S-box output. In this variant, they use a function called compare $_{b}(x, y)$, which returns $b$ if $x=y$ and $\bar{b}$ otherwise. A first-order secure implementation of compare $_{b}$ is necessary to guarantee the security of the algorithm. To this end, Rivain et al [21] presented a method for implementing the compare $_{b}$ function, shown in Algorithm 3. The secure S-box computation works as follows: First, a random bit $b$ is generated, which is one of the inputs to the compare $_{b}$ function. Then, for each possible value of $a$, the algorithm computes $\left(S\left(x_{1} \oplus a\right) \oplus y_{1}\right) \oplus y_{2}$, which will be written to either $R_{b}$ or $R_{\bar{b}}$, depending on

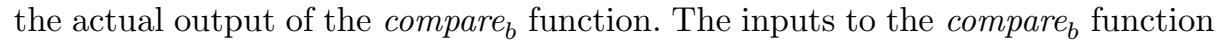
are $x_{2} \oplus a$ and $x_{3}$. When $a=x_{2} \oplus x_{3}$, compare $_{b}\left(x_{2} \oplus a, x_{3}\right)$ returns $b$, thus the result is stored in $R_{b}$. In all other cases, the returned value is $\bar{b}$, so the result is stored in the register $R_{\bar{b}}$. At the end of the algorithm, the value stored in $R_{b}$ is $S(x) \oplus y_{1} \oplus y_{2}$, which is exactly what we wanted to achieve.

Note that Algorithm 2 needs only $2^{n}$ bits in RAM, namely for the function compare $_{b}$. Thus, it requires $m$ times less memory than Algorithm 1, though the execution time is longer due to multiple calls to the compare $_{b}$ function.

\section{Applying Goubin's Conversion to Second Order}

In this section, we demonstrate that a straightforward application of Goubin's conversion technique [10] to the second order does not work. Assume we have 


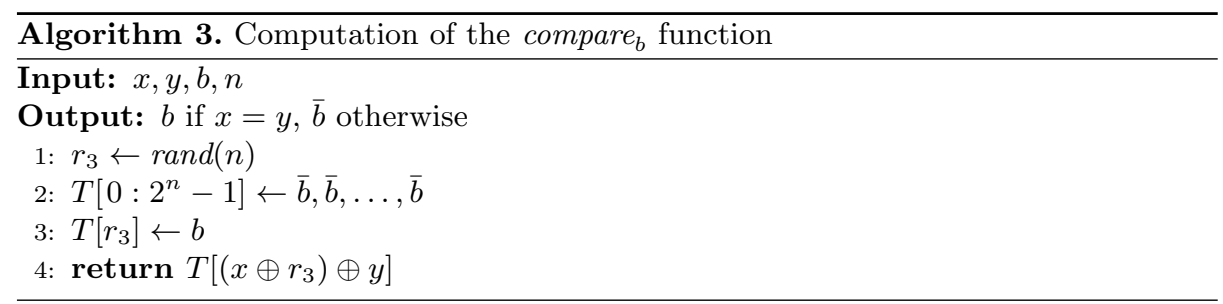

three Boolean shares $x_{1}, x_{2}, x_{3}$ whereby $x=x_{1} \oplus x_{2} \oplus x_{3}$. We need to find the arithmetic shares $A_{1}, A_{2}$, and $A_{3}$ such that $x=A_{1}+A_{2}+A_{3} \bmod 2^{n}$. To do so, we can iteratively compute $A_{1}, A_{2}, A_{3}$ as follows:

$$
\begin{aligned}
x & =A_{1}+\left(x_{2} \oplus x_{3}\right) \\
x & =A_{1}+A_{2}+x_{3} \\
A_{1} & =x-\left(x_{2} \oplus x_{3}\right) \\
A_{2} & =\left(x_{2} \oplus x_{3}\right)-x_{3} \\
A_{3} & =x_{3}
\end{aligned}
$$

Based on the above, we can compute $A_{1}$ in the following way:

$$
\begin{aligned}
A_{1} & =x_{1} \oplus\left(x_{2} \oplus x_{3}\right)-\left(x_{2} \oplus x_{3}\right)=\phi_{x_{1}}\left(x_{2} \oplus x_{3}\right) \\
& =\phi_{x_{1}}\left(x_{2}\right) \oplus \phi_{x_{1}}\left(x_{3}\right) \oplus x_{1} .
\end{aligned}
$$

One could try to securely compute $\phi_{x_{1}}\left(x_{2}\right)$ and $\phi_{x_{1}}\left(x_{3}\right)$ as follows:

$$
\begin{aligned}
& \phi_{x_{1}}\left(x_{2}\right)=\phi_{x_{1}}\left(x_{2} \oplus r\right) \oplus \phi_{x_{1}}(r) \oplus x_{1} \\
& \phi_{x_{1}}\left(x_{3}\right)=\phi_{x_{1}}\left(x_{3} \oplus r\right) \oplus \phi_{x_{1}}(r) \oplus x_{1} .
\end{aligned}
$$

This means,

$$
\begin{aligned}
A_{1} & =\phi_{x_{1}}\left(x_{2} \oplus r\right) \oplus \phi_{x_{1}}(r) \oplus \phi_{x_{1}}\left(x_{3} \oplus r\right) \oplus \phi_{x_{1}}(r) \oplus x_{1} \\
& =\phi_{x_{1}}\left(x_{2} \oplus r\right) \oplus \phi_{x_{1}}\left(x_{3} \oplus r\right) \oplus x_{1} \\
& =\left(\left(x_{1} \oplus x_{2} \oplus r\right)-\left(x_{2} \oplus r\right)\right) \oplus\left(\left(x_{1} \oplus x_{3} \oplus r\right)-\left(x_{3} \oplus r\right)\right) \oplus x_{1} .
\end{aligned}
$$

But we can combine the leakages from $x_{1} \oplus x_{2} \oplus r$ and $x_{3} \oplus r$ to get $x_{1} \oplus x_{2} \oplus$ $x_{3}=x$, inducing a second order attack. Similarly, we can combine the leakages from $x_{1} \oplus x_{3} \oplus r$ and $x_{2} \oplus r$ to get $x_{1} \oplus x_{2} \oplus x_{3}=x$. Now, let us consider the case where we use a different random $r_{i}$ for computing each $\phi_{x_{i}}\left(x_{j}\right)$, i.e.

$$
\begin{aligned}
& \phi_{x_{1}}\left(x_{2}\right)=\phi_{x_{1}}\left(x_{2} \oplus r_{1}\right) \oplus \phi_{x_{1}}\left(r_{1}\right) \oplus x_{1} \\
& \phi_{x_{1}}\left(x_{3}\right)=\phi_{x_{1}}\left(x_{3} \oplus r_{2}\right) \oplus \phi_{x_{1}}\left(r_{2}\right) \oplus x_{1} .
\end{aligned}
$$

This means,

$$
A_{1}=\phi_{x_{1}}\left(x_{2} \oplus r_{1}\right) \oplus \phi_{x_{1}}\left(r_{1}\right) \oplus \phi_{x_{1}}\left(x_{3} \oplus r_{2}\right) \oplus \phi_{x_{1}}\left(r_{2}\right) \oplus x_{1} .
$$


Now, when computing $A_{1}$, regardless of what sequence we choose, we would be leaking the secret $x$ while combining the results. For example, assume that we calculate according to the following sequence:

$$
\begin{aligned}
\phi_{x_{1}}\left(r_{1}\right) \oplus \phi_{x_{1}}\left(x_{3} \oplus r_{2}\right) \oplus \phi_{x_{1}}\left(r_{2}\right) & =\phi_{x_{1}}\left(x_{3} \oplus r_{1}\right) \\
& =\left(\left(x_{1} \oplus x_{3} \oplus r_{1}\right)-\left(x_{3} \oplus r_{1}\right)\right)
\end{aligned}
$$

Let us further assume that $\phi_{x_{1}}\left(x_{2} \oplus r_{1}\right)$ is calculated as follows:

$$
\phi_{x_{1}}\left(x_{2} \oplus r_{1}\right)=\left(\left(x_{1} \oplus x_{2} \oplus r_{1}\right)-\left(x_{2} \oplus r_{1}\right)\right)
$$

Then, we can combine the leakages from $x_{1} \oplus x_{2} \oplus r_{1}$ and $x_{3} \oplus r_{1}$ to find the value of $x$. From this, we conclude that the straightforward application of the method of Goubin does not work for second order.

\section{Second-Order Boolean to Arithmetic Masking}

This section addresses the problem of securely converting second-order Boolean shares to the corresponding arithmetic shares without any second-order or firstorder leakage. To start with, we are given three Boolean shares $x_{1}, x_{2}, x_{3}$ such that $x=x_{1} \oplus x_{2} \oplus x_{3}$ where $x$ is a sensitive variable. The goal is to find three arithmetic shares $A_{1}, A_{2}, A_{3}$ satisfying $x=A_{1}+A_{2}+A_{3}$ without leaking any information exploitable in a first or second-order DPA attack. We propose two algorithms to achieve this goal; one is based on Algorithm 1 and the second on Algorithm 2. Both of our algorithms use the secure S-box output computation of Rivain et al [21], which simplifies the security proofs.

The first of our variants is given in Algorithm 4; we devised this conversion by modifying Algorithm 1 appropriately. The algorithm generates two shares $A_{2}, A_{3}$ randomly from $\left[0,2^{n}-1\right]$ and computes the third share via the relation $A_{1}=\left(x-A_{2}\right)-A_{3}$. The aim of Algorithm 1 was to output $S(x) \oplus y_{1} \oplus y_{2}$ as result. Hence, it computed $\left(S\left(x_{1} \oplus a\right) \oplus y_{1}\right) \oplus y_{2}$ for every possible value of the variable $a$ from 0 to $2^{n}-1$, and then obtained the correct value for the case $a=x_{2} \oplus x_{3}$. But here, our aim is to compute $\left(x-A_{2}\right)-A_{3}$, which requires us to modify the table entries to $\left(\left(x_{1} \oplus a\right)-A_{2}\right)-A_{3}$ so that we can obtain the correct value when $a=x_{2} \oplus x_{3}$. Note that the subtractions are modulo $2^{n}$.

Correctness: When $a^{\prime}=r, a$ becomes $r \oplus r^{\prime}=x_{2} \oplus x_{3}$. Thus, $T\left[a^{\prime}\right]=T[r]=$ $\left(\left(\left(\left(x_{1} \oplus x_{2}\right) \oplus x_{3}\right)-A_{2}\right)-A_{3}\right)=\left(x-A_{2}\right)-A_{3}$, from which follows that $A_{1}=$ $\left(x-A_{2}\right)-A_{3}$ and finally $x=A_{1}+A_{2}+A_{3}$.

We devised Algorithm 5 by appropriately adapting Algorithm 2. Again, we first compute the value of $\left(\left(x_{1} \oplus a\right)-A_{2}\right)-A_{3}$ for all possible values of $a$ and store the result in $R_{b}$ or $R_{\bar{b}}$, depending on the return value of compare $_{b}$. When $a=x_{2} \oplus x_{3}$, the value of $x_{2} \oplus a$ and $x_{3}$ become equal, hence compare $e_{b}$ returns $b$. Consequently, the correct value of $A_{1}=\left(x-A_{2}\right)-A_{3}$ is stored in $R_{b}$. In all other cases (i.e. $\left.a \neq x_{2} \oplus x_{3}\right)$, the value $\left(\left(x_{1} \oplus a\right)-A_{2}\right)-A_{3}$ is stored in $R_{\bar{b}}$. 

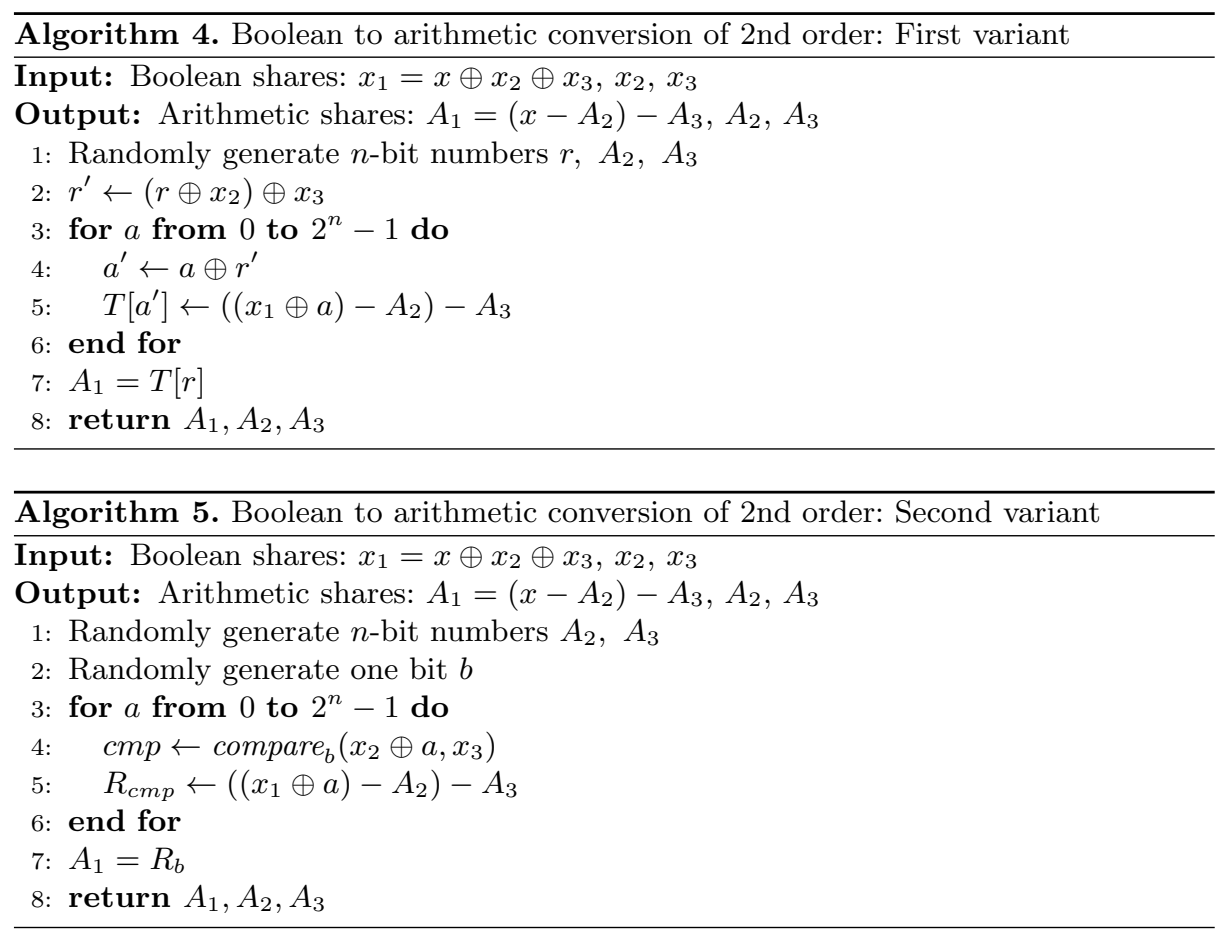

\section{Second-Order Arithmetic to Boolean Masking}

In this section, we briefly introduce two algorithms to securely convert secondorder arithmetic shares into the "corresponding" Boolean shares, whereby the conversion does not introduce any second-order (or first-order) leakage. More precisely, given three arithmetic shares $A_{1}, A_{2}, A_{3}$ of a sensitive variable $x$ such that $x=A_{1}+A_{2}+A_{3}$, both of these algorithms compute the Boolean shares $x_{1}, x_{2}, x_{3}$ satisfying $x=x_{1} \oplus x_{2} \oplus x_{3}$ without second or first-order leakage.

Algorithm 6 employs a lookup table similar to Algorithm 4. Here, the value of $r^{\prime}$ is $\left(A_{2}-r\right)+A_{3}$, where $r$ is a random value in the range $\left[0,2^{n}-1\right]$. The table entries corresponding to $a^{\prime}=a-r^{\prime}$ are now $\left((A 1+a) \oplus x_{2}\right) \oplus x_{3}$ instead of $\left(\left(x_{1} \oplus a\right)-A_{2}\right)-A_{3}$. Similar to Algorithm 4, the two shares $x_{2}$ and $x_{3}$ are generated randomly from $\left[0,2^{n}-1\right]$, while the third share $x_{1}$ is $T[r]$.

Correctness: When $a^{\prime}=r, a$ becomes $r+r^{\prime}=A_{2}+A_{3}$. Thus, $T\left[a^{\prime}\right]=T[r]=$ $\left.\left(\left(\left(A_{1}+A_{2}\right)+A_{3}\right) \oplus x_{2}\right) \oplus x_{3}\right)=\left(x \oplus x_{2}\right) \oplus x_{3}$, from which follows that $x_{1}=$ $\left(x \oplus x_{2}\right) \oplus x_{3}$ and finally $x=x_{1} \oplus x_{2} \oplus x_{3}$.

Algorithm 7 shows the other method to convert arithmetic shares of second order to "equivalent" Boolean shares. Among the three Boolean shares, $x_{2}$ and $x_{3}$ are generated randomly within the range $\left[0,2^{n-1}\right]$. One of the two registers $R_{0}, R_{1}$ serves to store the correct value of $x_{1}$ and the other is used for storing 

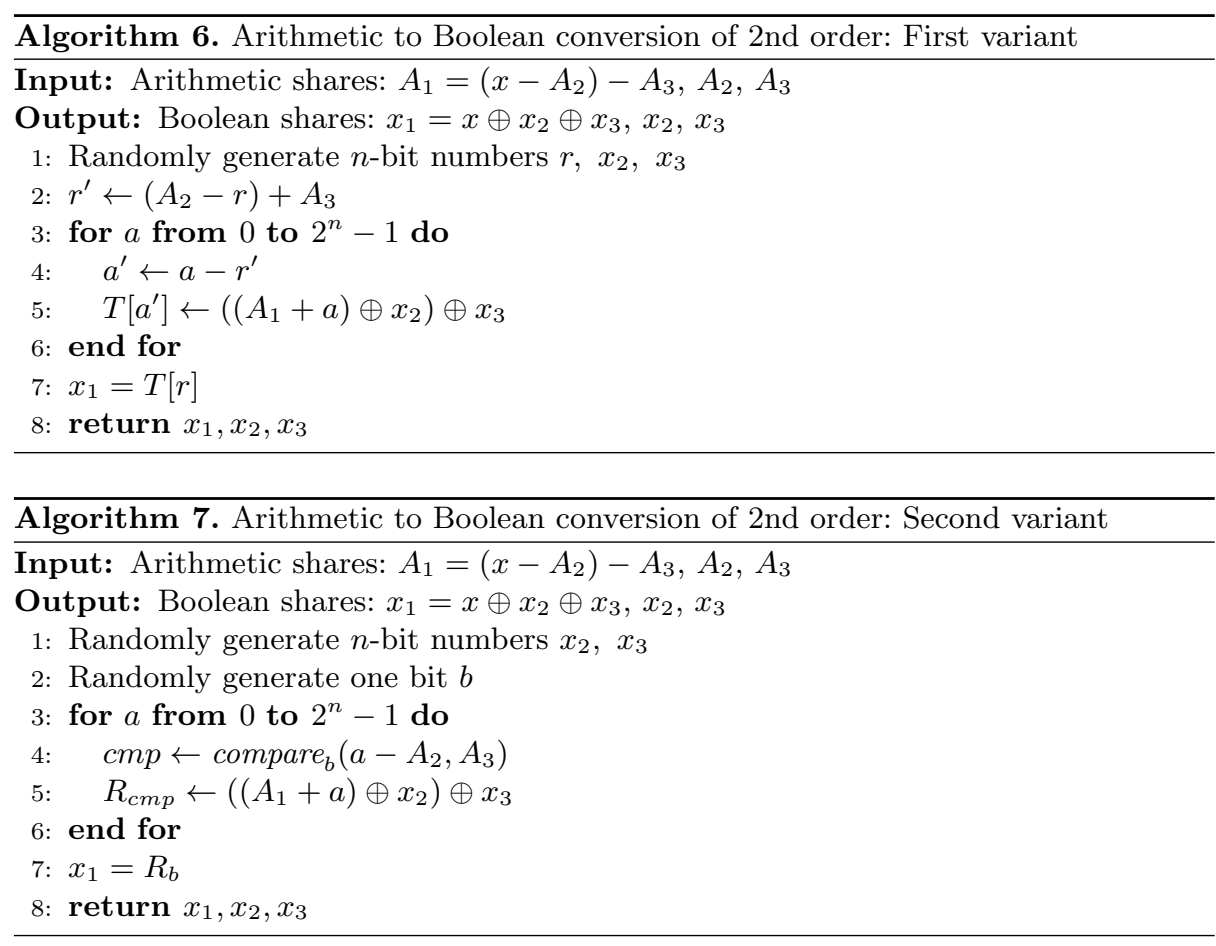

the incorrect value. The compare instruction compares $\left(a-A_{2}\right)$ with $A 3$; when they are equal, compare $e_{b}$ returns $b$ and, thus, the result is stored in $R_{b}$. In this case, the result is the correct value of $x_{1}$, which means $\left(\left(A_{1}+A_{2}+A_{3}\right) \oplus x_{2}\right) \oplus$ $x_{3}=\left(x \oplus x_{2}\right) \oplus x_{3}$. Otherwise, the result is incorrect and stored in $R_{b}^{\prime}$.

\section{Security Analysis}

We first review the security model introduced in [21]. Then, based on the same model, we present the security proofs of all our four algorithms against secondorder attacks. We assume that the device leaks in the Hamming weight model (i.e. the leakage is proportional to the Hamming weight of the data processed on the device). Below we summarize some basic definitions and results that are used in the proofs for quick reference (partly taken from [21]).

- Sensitive variable: An intermediate variable obtained by applying a certain function on a known value (e.g. plaintext) and the secret key.

- Primitive random variable: An intermediate variable generated by a random number generator with uniform distribution.

- Functional dependence: If an intermediate variable is obtained by applying a discrete function on some other variable $X$, then it is said to be functionally dependent on $X$. Otherwise, it is functionally independent. 
- Statistical dependence: If the statistical distribution of an intermediate variable varies according to some other variable $X$, then it is said to be statistically dependent on $X$. Otherwise, it is statistically independent.

- Functional independence implies statistical independence, but the converse is false.

- In second-order DPA, leakages from at most two intermediate variables are allowed to be exploited simultaneously. So, for a cryptographic algorithm to be called second-order secure, it is important that every pair of intermediate variables is statistically independent of any sensitive variable.

- A set of intermediate variables is statistically independent from a variable $X$ if, and only if, all intermediate variables belonging to the set are statistically independent of $X$.

- Given two sets $A$ and $B, A \times B$ is statistically independent from a variable $X$ if, and only if, all pairs in $A \times B$ are statistically independent of $X$.

- Lemma 1. For statistically independent random variables $X$ and $Y$, it holds that for every measurable function $f, f(X)$ is statistically independent of the variable $Y$.

- Lemma 2. Let $X$ and $Y$ be statistically independent random variables where $Y$ is uniformly distributed, and $Z$ a variable that is statistically independent of $Y$ and functionally independent of $X$. In this case, the pair $(Z, X \oplus Y)$ is statistically independent of $X$.

Limitations of the Security Proofs: The algorithms in [21], though proven secure against "standard" DPA attacks, suffer from two problems. Firstly, the algorithm not using table computations, i.e. Algorithm 2, is only secure in the Hamming weight model. At COSADE 2012, Coron et al have shown that this algorithm is not secure when the device leaks in the Hamming distance model [3]. They also demonstrated that a straightforward conversion of a proof from the Hamming weight model to Hamming distance model by initializing the bus (resp. register) with 0 before every write operation has a second-order flaw. As a consequence, the proof of Algorithm 2 from [21] is not valid anymore in the Hamming distance model. The conversion of a security proof from one leakage model to another is still an open issue. Since Algorithm 5 and Algorithm 7 are similar to Algorithm 2, they suffer from said limitation too. However, a solution to the conversion problem for Rivain et al's generic countermeasure for secure S-box computation would, of course, also apply to our algorithms.

Secondly, some current developments in side-channel cryptanalysis indicate that masking might succumb to a so-called horizontal side-channel attack (see e.g. $[20,22])$. By targeting the table generation phase of a masking scheme, an attacker may succeed to recover the secret key when the signal-to-noise ratio is low. However, these attacks are generic in the sense that they are applicable to essentially any practical masking scheme; our algorithms are no exception. The problem of securely generating the masked table is still an open challenge and requires further attention. Any solution to this problem can be readily applied to our algorithms as well to help them resist horizontal attacks. Hence, despite these limitations, our algorithms are still practically relevant. 
Proposition 1. Algorithm 4 is secure against second-order DPA.

Proof. We follow the notation of [21] for the sake of simplicity. Each intermediate variable of the algorithm can be seen as a result of applying the function $I_{j}$ on the loop index $a$. Assume that $I_{\text {index }}=I_{\text {index }}(a)$ for $0 \leq a \leq 2^{n}-1$ and $I=\bigcup_{\text {index }=0}^{\text {num }} I_{\text {index }}$, whereby num specifies the total number of intermediate variables. We list all intermediate variables used in Algorithm 4 in Table 1.

Table 1. Intermediate variables used in Algorithm 4

\begin{tabular}{|c|c|}
\hline index & $I_{\text {index }}$ \\
\hline 1 & $x_{2}$ \\
2 & $x_{3}$ \\
3 & $A_{2}$ \\
4 & $A_{3}$ \\
5 & $r$ \\
6 & $r \oplus x_{2}$ \\
7 & $r \oplus x_{2} \oplus x_{3}$ \\
8 & $a$ \\
9 & $a \oplus r \oplus x_{2} \oplus x_{3}$ \\
10 & $x \oplus x_{2} \oplus x_{3}$ \\
11 & $x \oplus x_{2} \oplus x_{3} \oplus a$ \\
12 & $\left(x \oplus x_{2} \oplus x_{3} \oplus a\right)-A_{2}$ \\
13 & $\left.\left(x \oplus x_{2} \oplus x_{3} \oplus a\right)-A_{2}\right)-A_{3}$ \\
14 & $\left(x-A_{2}\right)-A_{3}$ \\
\hline
\end{tabular}

Table 2. Intermediate variables used in Algorithm 5

\begin{tabular}{|c|c|}
\hline index & $I_{\text {index }}$ \\
\hline 1 & $x_{2}$ \\
2 & $x_{3}$ \\
3 & $A_{2}$ \\
4 & $A_{3}$ \\
5 & $b$ \\
6 & $a$ \\
7 & $x_{2} \oplus a$ \\
8 & $\delta_{0}\left(x_{2} \oplus a \oplus x_{3}\right) \oplus b$ \\
9 & $x \oplus x_{2} \oplus x_{3}$ \\
10 & $x \oplus x_{2} \oplus x_{3} \oplus a$ \\
11 & $\left(x \oplus x_{2} \oplus x_{3} \oplus a\right)-A_{2}$ \\
12 & $\left(\left(x \oplus x_{2} \oplus x_{3} \oplus a\right)-A_{2}\right)-A_{3}$ \\
13 & $\left(x-A_{2}\right)-A_{3}$ \\
\hline
\end{tabular}

We recall that, for an algorithm to be secure against second-order DPA, no pair of intermediate variables should be statistically dependent on a sensitive variable. Consequently, we need to prove that $I \times I$ is statistically independent of $x$. To simplify matters, we divide the set of intermediate variables into three subsets: $E_{1}=I_{1} \cup I_{2} \cup \ldots \cup I_{9}, E_{2}=I_{10} \cup I_{11} \cup \ldots \cup I_{13}$, and $E_{3}=I_{14}$. The objective now is to prove that all possible combinations of these three sets are statistically independent of $x$.

1. $\mathbf{E}_{\mathbf{1}} \times \mathbf{E}_{\mathbf{1}}$ : All the intermediate variables in $E_{1}$ are functionally independent of $x$. Hence, $E_{1} \times E_{1}$ is statistically independent of $x$.

2. $\mathbf{E}_{\mathbf{2}} \times \mathbf{E}_{\mathbf{2}}$ : It can be seen that $I_{10}=x \oplus x_{2} \oplus x_{3}$ is statistically independent of $x$. As all elements in $E_{2} \times E_{2}$ are functions of $I_{10}$, it can be inferred that $E_{2} \times E_{2}$ is statistically independent of $x$ by applying Lemma 2 .

3. $\mathbf{E}_{\mathbf{3}} \times \mathbf{E}_{3}$ : It is also straightforward to see that $E_{3} \times E_{3}$ is statistically independent of $x$ since $x-\left(A_{2}-A_{3}\right)$ is statistically independent of $x$.

4. $\mathbf{E}_{\mathbf{1}} \times \mathbf{E}_{\mathbf{2}}: E_{1}$ is statistically independent of $\left(x_{2} \oplus x_{3}\right)$ and functionally independent of $x$. According to Lemma $2, E_{1} \times\left\{x \oplus x_{2} \oplus x_{3}\right\}$ is statistically independent of $x$. Hence, according to Lemma $1, E_{1} \times E_{2}$ is statistically independent of $x$. 
5. $\mathbf{E}_{1} \times \mathbf{E}_{3}$ : Since $E_{1}$ is statistically independent of $A_{2}-A_{3}$, the combination $E_{1} \times\left\{x-\left(A_{2}-A_{3}\right)\right\}$ (i.e. $\left.E_{1} \times E_{3}\right)$ is statistically independent of $x$. As the pair $\left(x \oplus x_{2} \oplus x_{3},\left(x-A_{2}\right)-A_{3}\right)$ is statistically independent of $x$, it holds that $\left(I_{10} \cup I_{11} \cup I_{12}\right) \times E_{3}$ is statistically independent of $x$ because all these can be expressed as a function of $\left(x \oplus x_{2} \oplus x_{3},\left(x-A_{2}\right)-A_{3}\right)$.

6. $\mathbf{E}_{\mathbf{2}} \times \mathbf{E}_{\mathbf{3}}$ : We need to prove that $I_{13} \times E_{3}$ is statistically independent of $x$ to establish that $E_{2} \times E_{3}$ is statistically independent of $x$. Suppose that $v_{1}=$ $\left(x-A_{2}\right)-A_{3}$ and $v_{2}=\left(x \oplus x_{2} \oplus x_{3} \oplus a\right)$. It can be seen that $v_{1}$ and $v_{2}$ are statistically independent of each other as well as of variable $x$. We can write $I_{13} \times E_{3}$ as $\left\{v_{1}+v_{2}-x\right\} \times v_{1}$, which is statistically independent of $x$.

From all this it can be concluded that Proposition 1 holds.

Proposition 2. Algorithm 5 is secure against second-order DPA.

Proof. Assume that the Boolean function $\delta_{0}(x)=0$ only when $x=0$. So, the function $\operatorname{compare}_{b}(x, y)$ can be represented as $\delta_{0}(x \oplus y) \oplus b$. For Algorithm 5 to be secure, it is important that the compare $_{b}$ function is implemented in a way which prevents any first-order leakage on compare $(x, y)$. One such method is recalled in Algorithm 3 (originally proposed in [21]) and we can construct the proof on this method. The intermediate variables appearing in Algorithm 5 are given in Table 2 . It can be easily seen that nearly all intermediate variables are identical to those in Algorithm 4. Thus, we can prove the security of Algorithm 5 by using the same arguments as given in the proof of Proposition 1.

Table 3. Intermediate variables used in Algorithm 6

\begin{tabular}{|c|c|}
\hline index & $I_{\text {index }}$ \\
\hline 1 & $x_{2}$ \\
2 & $x_{3}$ \\
3 & $A_{2}$ \\
4 & $A_{3}$ \\
5 & $r$ \\
6 & $A_{2}-r$ \\
7 & $A_{2}-r+A_{3}$ \\
8 & $a$ \\
9 & $a-A_{2}+r-A_{3}$ \\
10 & $x-A_{2}-A_{3}$ \\
11 & $x-A_{2}-A_{3}+a$ \\
12 & $\left(x-A_{2}-A_{3}+a\right) \oplus x_{2}$ \\
13 & $\left(x-A_{2}-A_{3}+a\right) \oplus x_{2} \oplus x_{3}$ \\
14 & $x \oplus x_{2} \oplus x_{3}$ \\
\hline
\end{tabular}

Table 4. Intermediate variables used in Algorithm 7

\begin{tabular}{|c|c|}
\hline index & $I_{\text {index }}$ \\
\hline 1 & $x_{2}$ \\
2 & $x_{3}$ \\
3 & $A_{2}$ \\
4 & $A_{3}$ \\
5 & $b$ \\
6 & $a$ \\
7 & $a-A_{2}$ \\
8 & $\delta_{0}\left(\left(a-A_{2}\right) \oplus A_{3}\right) \oplus b$ \\
9 & $x-A_{2}-A_{3}$ \\
10 & $x-A_{2}-A_{3}+a$ \\
11 & $\left(x-A_{2}-A_{3}+a\right) \oplus x_{2}$ \\
12 & $\left(x-A_{2}-A_{3}+a\right) \oplus x_{2} \oplus x_{3}$ \\
13 & $x \oplus x_{2} \oplus x_{3}$ \\
\hline
\end{tabular}

Proposition 3. Algorithm 6 is secure against second-order DPA. 
Proof. Table 3 lists all intermediate variables appearing in Algorithm 6. We can use similar arguments as in Proposition 1 to prove that no pair of intermediate variables is statistically dependent on $x$.

Proposition 4. Algorithm 7 is secure against second-order DPA.

Proof. We show all intermediate variables that appear in Algorithm 7 in Table 4. Again, the security proof can be developed similar to the one of Proposition 1 , namely by showing that no pair of the intermediate variables is statistically dependent on $x$.

\section{Implementation Results}

We implemented all algorithms from Section 4 and Section 5 in Matlab and in ANSI C, whereby we only considered the simplest case of converting between 8-bit masks. The Matlab implementation served as a reference for the $\mathrm{C}$ implementation so that we could easily verify the correctness of the latter. We tested our four algorithms individually using 100000 pseudo-random inputs and found that all of them produce the correct result in all cases. The $\mathrm{C}$ implementation generates the random numbers with help of the rand function of the standard C library ${ }^{1}$. Although this is sufficient for testing, a real-world implementation would need pseudo-random numbers of better quality. Furthermore, it should be noted that we developed all our implementations primarily for the purpose of having a proof of concept rather than achieving high performance. The implementations can be further optimized, which means the results we report in this section should be seen as upper bounds of the execution time. Also, if the conversions are used in real-world applications, one needs to take care that the compilation process respects the flow of intermediate variables assumed in the security proofs given in Section 6. If this is not the case, it becomes necessary to develop an assembly language implementation.

The implementation of Algorithm 4 and Algorithm 6 is straightforward; we create a table of 256 bytes and, for each of the 256 possible values of $a$, store the corresponding entry in a byte. The indexing of the table is done via $a^{\prime}$ and the correct value of the third share is retrieved by accessing the table entry corresponding to $r$. An optimized implementation of Algorithm 5 and Algorithm 7 has to perform the compare $_{b}$ function as efficiently as possible. We used the following approach to implement this function. First, we create an array of 32 bytes and initialize all the bits to $\bar{b}$. We treat the array as a collection of 256 bits, all initialized to $\bar{b}$. Then, for a random value $r_{3}$, we set the corresponding bit position in the array to $b$. Each call to compare $_{b}$ is now simply replaced by a single look-up into the array. To give an example, $\operatorname{compare}_{b}(x, y)$ is obtained by retrieving the value at the bit position $\left(x \oplus r_{3}\right) \oplus y$. The index of the byte containing the bit can be obtained through a logical right-shift operation. The bit itself can be extracted from the byte via a shift operation too.

\footnotetext{
${ }^{1}$ On an 8-bit AVR processor, e.g. ATmega128, calling the rand function takes some 800 clock cycles when using the avr-libc library of the WinAVR tool suite.
} 
Table 5. Implementation results on an 8, 16 and 32-bit platform

\begin{tabular}{|c|c|c|}
\hline Algorithm & Cycles & RAM (bytes) \\
\hline \multicolumn{3}{|c|}{ 8-bit architecture (AVR) } \\
\hline Algorithm 4 & 5769 & 256 \\
\hline Algorithm 5 & 6742 & 32 \\
\hline Algorithm 6 & 5769 & 256 \\
\hline Algorithm 7 & 6742 & 32 \\
\hline \multicolumn{3}{|c|}{ 16-bit architecture (MSP) } \\
\hline Algorithm 4 & 4983 & 256 \\
\hline Algorithm 5 & 16706 & 32 \\
\hline Algorithm 6 & 4983 & 256 \\
\hline Algorithm 7 & 16706 & 32 \\
\hline \multicolumn{3}{|c|}{ 32-bit architecture (ARM) } \\
\hline Algorithm 4 & 793 & 256 \\
\hline Algorithm 5 & 1087 & 32 \\
\hline Algorithm 6 & 793 & 256 \\
\hline Algorithm 7 & 1087 & 32 \\
\hline
\end{tabular}

In order to assess the execution time of the algorithms, we compiled them for the 32-bit ARM platform as well as the 8-bit AVR platform and performed simulations with AVR Studio. In addition, we evaluated our four algorithms on a low-power 16-bit micro-controller, the TI MSP430, with the help of a cycleaccurate instruction-set simulator. Table 5 illustrates the simulation results we obtained on these three platforms. The second column of Table 5 specifies the execution time (in clock cycles) needed to convert an 8-bit mask from one form to the other. The third column gives the memory (RAM) requirements of the algorithms in number of bytes. As we can see, the two algorithms using table look-ups, i.e. Algorithm 4 and Algorithm 6, are faster than the ones which do not use tables. This is because of the additional time required to evaluate the compare $_{b}$ function in the case of Algorithm 5 and Algorithm 7. However, both algorithms based on the table computation method require exactly eight times more memory than their counterparts.

Note that the execution times of Algorithm 5 and Algorithm 7 obtained on the 16-bit platform are somewhat misleading. As can be seen from Table 5 , the execution time on the TI MSP430 is by a factor of roughly 2.5 slower than the time on the 8-bit AVR. This can be explained through the fact that the used MSP430 processor does not have a barrel shifter, which means a shift operation by $n$ bit positions takes $n$ clock cycles. On the other hand, the AVR features a fast barrel shifter able to execute all shift operations in one cycle, irrespective of the shift distance.

\section{Conclusions}

In this paper, we addressed the practical problem of converting between secondorder Boolean and arithmetic masking. We introduced two algorithms secure 
against second-order attacks for each direction by applying the generic secondorder secure countermeasure proposed by Rivain et al at FSE 2008. The time complexity of these algorithms is $O\left(2^{n}\right)$, where $n$ is the size of the data to be converted. All algorithms are proven to be secure when the device leaks in the Hamming weight model. Our implementation results show that the algorithms without tables require eight times less memory (i.e. RAM) than the table-based algorithms, but the saving in RAM footprint comes at the expense of increased execution time. The proposed algorithms become costly when the length of the data to be converted exceeds 16 bits. As part of our future research, we aim to improve the efficiency of the conversion methods by devising algorithms with a better time-memory trade-off.

\section{References}

1. D. Agrawal, B. Archambeault, J. R. Rao, and P. Rohatgi. The EM side-channel(s). In Cryptographic Hardware and Embedded Systems - CHES 2002, vol. 2523 of Lecture Notes in Computer Science, pp. 29-45. Springer Verlag, 2003.

2. S. Chari, C. S. Jutla, J. R. Rao, and P. Rohatgi. Towards sound approaches to counteract power-analysis attacks. Advances in Cryptology - CRYPTO '99, vol. 1666 of Lecture Notes in Computer Science, pp. 398-412. Springer Verlag, 1999.

3. J.-S. Coron, C. Giraud, E. Prouff, S. Renner, M. Rivain, and P. K. Vadnala. Conversion of security proofs from one leakage model to another: A new issue. In Constructive Side-Channel Analysis and Secure Design - COSADE 2012, vol. 7275 of Lecture Notes in Computer Science, pp. 69-81. Springer Verlag, 2012.

4. J.-S. Coron and L. Goubin. On Boolean and arithmetic masking against differential power analysis. In Cryptographic Hardware and Embedded Systems - CHES 2000, vol. 1965 of Lecture Notes in Computer Science, pp. 231-237. Springer Verlag, 2000.

5. J.-S. Coron and A. Tchulkine. A new algorithm for switching from arithmetic to Boolean masking. In Cryptographic Hardware and Embedded Systems - CHES 2003, vol. 2779 of Lecture Notes in Computer Science, pp. 89-97. Springer Verlag, 2003.

6. B. Debraize. Efficient and provably secure methods for switching from arithmetic to Boolean masking. In Cryptographic Hardware and Embedded Systems - CHES 2012, vol. 7428 of Lecture Notes in Computer Science, pp. 107-121. Springer Verlag, 2012.

7. L. Genelle, E. Prouff, and M. Quisquater. Secure multiplicative masking of power functions. In Applied Cryptography and Network Security - ACNS 2010, vol. 6123 of Lecture Notes in Computer Science, pp. 200-217. Springer Verlag, 2010.

8. L. Genelle, E. Prouff, and M. Quisquater. Montgomery's trick and fast implementation of masked AES. In Progress in Cryptology - AFRICACRYPT 2011, vol. 6737 of Lecture Notes in Computer Science, pp. 153-169. Springer Verlag, 2011.

9. L. Genelle, E. Prouff, and M. Quisquater. Thwarting higher-order side channel analysis with additive and multiplicative maskings. In Cryptographic Hardware and Embedded Systems - CHES 2011, vol. 6917 of Lecture Notes in Computer Science, pp. 240-255. Springer Verlag, 2011.

10. L. Goubin. A sound method for switching between Boolean and arithmetic masking. In Cryptographic Hardware and Embedded Systems - CHES 2001, vol. 2162 of Lecture Notes in Computer Science, pp. 3-15. Springer Verlag, 2001. 
11. H. Handschuh and H. M. Heys. A timing attack on RC5. In Selected Areas in Cryptography - SAC '98, vol. 1556 of Lecture Notes in Computer Science, pp. 306-318. Springer Verlag, 1999.

12. J. Kelsey, B. Schneier, D. Wagner, and C. Hall. Side channel cryptanalysis of product ciphers. In Computer Security - ESORICS '98, vol. 1485 of Lecture Notes in Computer Science, pp. 97-110. Springer Verlag, 1998.

13. P. Kocher. Timing attacks on implementations of Diffie-Hellman, RSA, DSS, and other systems. In Advances in Cryptology - CRYPTO '96, vol. 1109 of Lecture Notes in Computer Science, pp. 104-113. Springer Verlag, 1996.

14. P. Kocher, J. Jaffe, and B. Jun. Differential power analysis. In Advances in Cryptology - CRYPTO '99, vol. 1666 of Lecture Notes in Computer Science, pp. 388-397. Springer Verlag, 1999.

15. S. Mangard, M. E. Oswald, and T. Popp. Power Analysis Attacks: Revealing the Secrets of Smart Cards. Springer Verlag, 2007.

16. T. S. Messerges. Using second-order power analysis to attack DPA resistant software. In Cryptographic Hardware and Embedded Systems - CHES 2000, vol. 1965 of Lecture Notes in Computer Science, pp. 238-251. Springer Verlag, 2000.

17. T. S. Messerges. Securing the AES finalists against power analysis attacks. In Fast Software Encryption - FSE 2000, vol. 1978 of Lecture Notes in Computer Science, pp. 150-164. Springer Verlag, 2001.

18. O. Neiße and J. Pulkus. Switching blindings with a view towards IDEA. In Cryptographic Hardware and Embedded Systems - CHES 2004, vol. 3156 of Lecture Notes in Computer Science, pp. 230-239. Springer Verlag, 2004.

19. E. Oswald, S. Mangard, C. Herbst, and S. Tillich. Practical second-order DPA attacks for masked smart card implementations of block ciphers. In Topics in Cryptology - CT-RSA 2006, vol. 3860 of Lecture Notes in Computer Science, pp. 192-207. Springer Verlag, 2006.

20. J. Pan, J. I. Hartog, and J. Lu. You cannot hide behind the mask: Power analysis on a provably secure $S$-box implementation. In Information Security Applications - WISA 2009, vol. 5932 of Lecture Notes in Computer Science, pp. 178-192. Springer Verlag, 2009.

21. M. Rivain, E. Dottax, and E. Prouff. Block ciphers implementations provably secure against second order side channel analysis. In Fast Software Encryption FSE 2008, vol. 5086 of Lecture Notes in Computer Science, pp. 127-143. Springer Verlag, 2008.

22. M. Tunstall, C. Whitnall, and E. Oswald. Masking tables-An underestimated security risk. In Fast Software Encryption - FSE 2013, vol. 8424 of Lecture Notes in Computer Science, pp. 425-444. Springer Verlag, 2014. 